\title{
An Evaluation of the Design and Usability of a Novel Robotic Bilateral Arm Rehabilitation Device for Patients with Stroke
}

\author{
Yu-Cheng Pei ${ }^{1,2,3,4}$, Jean-Lon Chen ${ }^{1,2}$, Alice M. K. Wong ${ }^{1,2 *}$ and Kevin C. Tseng ${ }^{1,3,5,6 *}$ \\ 'Department of Physical Medicine and Rehabilitation, Chang Gung Memorial Hospital at Taoyuan, Taoyuan, Taiwan, ${ }^{2}$ School \\ of Medicine, Chang Gung University, Taoyuan, Taiwan, ${ }^{3}$ Healthy Aging Research Center, Chang Gung University, Taoyuan, \\ Taiwan, ${ }^{4}$ Center for Vascularized Composite Allotransplantation, Chang Gung Memorial Hospital at Linkou, Taoyuan, Taiwan, \\ ${ }^{5}$ Product Design and Development Laboratory, Department of Industrial Design, College of Management, Chang Gung \\ University, Taoyuan, Taiwan, ${ }^{6}$ Department of Design, National Taiwan Normal University, Taipei City, Taiwan
}

Study design: Case series.

Evidence level: IV (case series).

Introduction: Robot-assisted therapy for upper limb rehabilitation is an emerging research topic and its design process must integrate engineering, neurological pathophysiology, and clinical needs.

\section{OPEN ACCESS}

Edited by:

Ganesh R. Naik,

Western Sydney University, Australia

Reviewed by:

Frederick Robert Carrick, Bedfordshire Centre for Mental Health Research in Association with University of Cambridge,

United Kingdom Andrej Gams, Jozef Stefan Institute, Slovenia

*Correspondence: Alice M. K. Wong walice@adm.cgmh.org.tw; Kevin C. Tseng ktseng@pddlab.org

Received: 11 February 2017 Accepted: 11 July 2017

Published: 28 July 2017

Citation:

Pei Y-C, Chen J-L, Wong AMK and Tseng KC (2017) An Evaluation of the

Design and Usability of a Novel Robotic Bilateral Arm Rehabilitation Device for Patients with Stroke. Front. Neurorobot. 11:36. doi: 10.3389/fnbot.2017.00036
Purpose of the study: This study developed/evaluated the usefulness of a novel rehabilitation device, the MirrorPath, designed for the upper limb rehabilitation of patients with hemiplegic stroke.

Methods: The process follows Tseng's methodology for innovative product design and development, namely two stages, device development and usability assessment. During the development process, the design was guided by patients' rehabilitation needs as defined by patients and their therapists. The design applied synchronic movement of the bilateral upper limbs, an approach that is compatible with the bilateral movement therapy and proprioceptive neuromuscular facilitation theories. MirrorPath consists of a robotic device that guides upper limb movement linked to a control module containing software controlling the robotic movement.

Results: Five healthy subjects were recruited in the pretest, and 4 patients, 4 caregivers, and 4 therapists were recruited in the formal test for usability. All recruited subjects were allocated to the test group, completed the evaluation, and their data were all analyzed. The total system usability scale score obtained from the patients, caregivers, and therapists was $71.8 \pm 11.9$, indicating a high level of usability and product acceptance.

Discussion and conclusion: Following a standard development process, we could yield a design that meets clinical needs. This low-cost device provides a feasible platform for carrying out robot-assisted bilateral movement therapy of patients with hemiplegic stroke.

Clinical Trial Registration: identifier NCT02698605.

Keywords: upper limb rehabilitation, stroke, rehabilitation device, usability, bilateral movement therapy 


\section{INTRODUCTION}

The World Health Organization (WHO) has reported that stroke is the third leading cause of death in developed countries and involves approximately 15 million stoke events annually. Onethird of stroke patients die and a further one-third of events results in permanent disability. Depending on the location of the brain insult, stroke can lead to a wide range of functional impairments (Mackay et al., 2004); these include language, cognition, sensation, and motor functions. Motor impairment impacts the patient's ability to perform activities of daily living. For the majority of patients, recovery of motor function involving an upper limb is slower than that of lower limb (Feys et al., 1998). Indeed, most activities of daily living rely the functioning of the upper limb, thus emphasizing the need for effective upper limb rehabilitation.

With an attempt to enhance the effectiveness of upper limb rehabilitation among stroke patients, a series of rehabilitation techniques have been developed and refined in recent decades; these include task-oriented motor training, constraint-induced movement therapy, mirror therapy, and bilateral movement training. Each of these methods has a number of theoretical advocates and each has been shown to be effective clinically. For instance, bilateral movement therapy, which involves coordinated movement of the bilateral upper limbs, has been shown to enhance upper limb recovery and coordination between the hands. Stoykov et al. (2009) found that bilateral arm training is more effective than unilateral training when restoring proximal upper limb function because it seems to improve the functional linkages between the bilateral hemispheres.

Even after receiving a full course of conventional rehabilitation, $60 \%$ of stroke patients still have difficulties when using their affected upper limb (Kwakkel et al., 1999). As a result, it has become the upmost importance to develop novel rehabilitation strategies that are able to help patients reach a higher level of recovery. One such approach is robot-assisted rehabilitation, which incorporates robotic technologies into the rehabilitation processes. Several well-known robot-assisted movement therapies for the upper limb has been implemented clinically, including MIT-Manus (Krebs et al., 1998), Bi-Manu-Track (Hesse et al., 2003), BATRAC (Cauraugh et al., 2010), and MIME (Burgar et al., 2000), each of which follows different movement therapy theories. Regarding the body parts that are mainly involved in therapy, Bi-Manu-Track focuses on the bilateral forearms and wrists, while BATRAC and MIME focus on the shoulder and elbow of the affected limb. Regarding the movement dimension, BATRAC involves movement in one-dimension, while MIME allows three-dimensional movement. In fact, the higher the degrees of freedom adopted during the movement therapy, the more complex is the design of the robotic device. As a result, it has become important to come up with a feasible design that fulfills the patient's rehabilitation needs while avoiding the high costs that can be associated with instrument acquirement and maintenance. Furthermore, the effectiveness of the system needs to be comparable to that provided by conventional therapies so that a motivation to pursue this therapeutic option can be established (Kwakkel et al., 2008; Lo et al., 2010).
As an approach to the development of mechanical rehabilitation devices for hemiplegic upper limbs, Timmermans et al. (2009) proposed that three design domains are required; these were the therapy techniques used, the motivation of the patient, and resulting performance rewards. An online survey of physical therapists, 233 in total, indicated that a preferred upper limb robotic device needs to accommodate different hand movements, to be able to be used while in a seated position, to be able to provide the user with feedback, to focus on the restoration of activities of daily living, to able to be used at home, to have adjustable resistance levels and to cost less than US\$6,000 (Lu et al., 2011).

In terms of usability, the interaction between the user and the machine tends to be overlooked during the development stage. Although a variety of upper limb rehabilitation machines have been proposed, only a few have been commercialized. This low market acceptance can be attributed to the high cost of these devices, safety concerns, and poor usability (Lee et al., 2005). To this end, the aim of this study was to design a bilateral upper limb rehabilitation device called MirrorPath for the rehabilitation of stroke patients that follows the theories of bilateral movement therapy and proprioceptive neuromuscular facilitation (PNF). These two theories were initially developed by Knott and Kabat and have been shown to have a positive effect on the range of active and passive motions needed by stroke patients (Sharman et al., 2006). Our device will guide the patient's upper limbs, each of which moves along a diagonal motion path on the horizontal plane. The position and velocity of motion of the bilateral limbs are perfectly mirrored across the midline on the table. Finally, usability testing was conducted on the completed prototype.

\section{MATERIALS AND METHODS}

This study adopts Tseng's methodology (Tseng, 2013) for innovative product design and development. This consists of two stages: (1) the device development and (2) usability assessment stages.

\section{Stage 1: Device Development}

This study began with observations at the Rehabilitation Center at Chang Gung Memorial Hospital, Taoyuan, Taiwan, targeting rehabilitation activities, and carrying out interviews of stroke patients and their therapists; this is because device development must be prefaced by an understanding of the users' needs. In order to allow patient privacy, non-participant observation techniques were used, with a focus on manual pen-and-paper observations. In addition, interviews were conducted with an occupational therapist and a physical therapist. To better understand patients who were potential device users, personal and situational analyses were used to explore potential product usage issues and to integrate the observation and interview results into the design process.

It was concluded that the ability to perform the rehabilitation movements needed to be the primary design consideration. The primary design consideration then determines future design factors, such as the appropriate movement mechanisms to be used, the choice of motors, and various ergonomic considerations. In this study, we have emphasized the rehabilitation of the 
proximal upper limbs and, therefore, any systems need to focus on shoulder and elbow movements. The motor that drives the patient's arm movement, therefore, needs to exceed the maximal mechanical loading predicted by the weight and resistance of the upper limb and the friction force of the apparatus. According to the 2005-2008, National Nutrition and Health Survey, the average weight of adult males and females in Taiwan for this period were 69.6 and $58.0 \mathrm{~kg}$, respectively. The weight of an individual's arm was then estimated as a proportion of total mass using gamma-ray measurements, which arrived at the average weights of single arms of $3.7 \mathrm{~kg}(36 \mathrm{~N})$ and $2.9 \mathrm{~kg}(28 \mathrm{~N})$ for adult males and females, respectively. In that, an additional safety factor was added to give a total estimated mechanical load of $54 \mathrm{~N}$. The device's dimensions were designed to accommodate up to the 95th percentile of the height of female adults aged 45-65 years old. Specifically, in this case, the 95th percentile of female adult was a shoulder-to-radial-styloid distance of $439 \mathrm{~mm}$.

\section{Stage 2: Usability Assessment}

This study protocol was specifically approved by the Institutional Review Board of Chang Gung Medical Foundation with ID 1015038A3. The study has been registered in https:/ClinicalTrials. gov with registration number NCT02698605. The usability assessment focused on the actual use of the proposed rehabilitation system in order to clarify the issues that users might face during actual system operation, which would provide a reference for subsequent system improvement. Pretesting and formal testing were conducted at Chang Gung Memorial Hospital, Taoyuan, Taiwan, a tertiary hospital that provide comprehensive neurorehabilitation. The pretesting was recruited through selfselection and formal testing through referral. The pretesting was conducted on five healthy participants, while the formal testing was conducted on 12 individuals (four stroke patients, four caregivers, and four therapists). The sample size was chosen according to the suggestion of Lewis and Sauro (2009), as a subject number of 12 was considered adequate for usability analyses. The healthy participants were aged 20-70 years old and had no physical disabilities; the stroke patients had normal cognitive and language skills, a stable stroke status, no fractures affecting the upper limbs during the previous 3 months, and minimal or no upper limb spasticity, namely a Modified Ashworth Scale of 0 or 1 . The occupational therapists had work experience in the hospital for more than 1 year.

This study was a one-arm study and all subjects used the device and received usability test. The study was conducted by research assistants. Each subject received one assessment that lasted $30 \mathrm{~min}$. The subjects were not paid for participation. In that, no randomization or masking was performed. Prior to the experiment part of the study, the subjects provided basic biographical information. We then explained the experimental process and demonstrated the operation of the device. For usability assessment, the subject operated the device under the instruction of the researchers, during which problems were identified and any questions asked by the participants recorded. The primary outcome was the system usability scale (SUS) questionnaire and the secondary outcomes were observational recording forms and open questions the subject reported during the assessment.
Following device operation, the subjects completed the SUS questionnaire that collected subjective evaluations and recommendations regarding the device. The experimental instruments used in this part of the study consisted of the rehabilitation device, video cameras, still image cameras, digital voice recorders, questionnaires, and observational recording forms (Figure 1).

The SUS was developed by Brooke (1996) as a system usability tool, which has been widely used in the evaluation of a range of systems (Brooke, 2013). The results of the SUS questionnaire were analyzed using SPSS. The split-half method was used for the reliability analysis and the one-sample $t$-test was used for statistical comparisons.

\section{RESULTS}

\section{Stage 1: Device Development Observations and Interviews}

The observation of patients with a need for upper limb rehabilitation showed the following clinical problems: (1) the patients had insufficient upper limb strength, (2) the patients had insufficient motivation to carry out autonomous rehabilitation, (3) the patients needed resistance adjustment to be available as part of the rehabilitation device, (4) the patients were insufficiently aware of their own rehabilitation status, and (5) the available therapistto-patient ratio is low and this would result in a situation where rehabilitation is unsupervised from time to time.

The results led to the following clinical recommendations: (1) due to the lack of therapist manpower, therapists may need

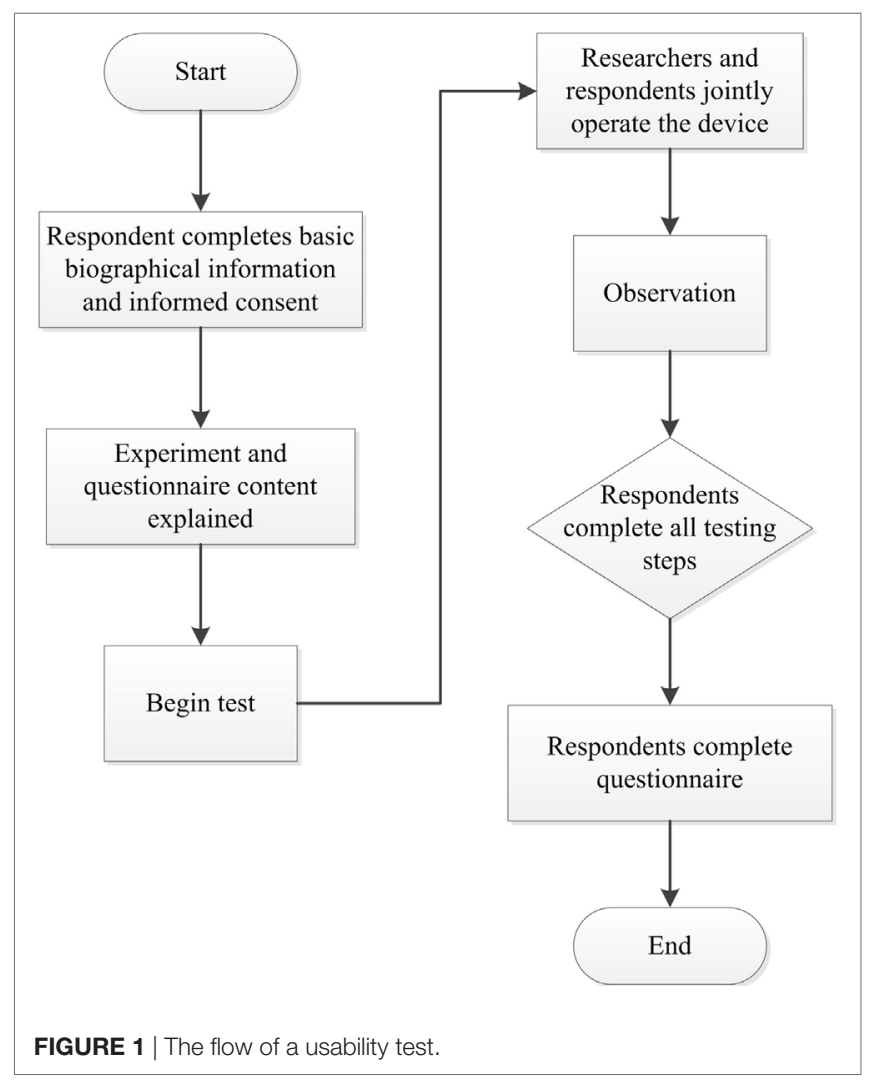


to supervise more than one patient at a time, (2) additional approaches are needed to encourage patients to achieve rehabilitation goals, (3) rehabilitation goal setting is highly reliant on patient intention including the extent of the exercise carried out, their learning status, their willingness to engage in rehabilitation, and (4) the design of the arm rehabilitation activities needs to be largely determined by the materials and equipment available to the therapist. The recommendations for the rehabilitation operation are that: (1) the use of fixed paths will be able to help the patient complete the rehabilitation exercise more smoothly and (2) the device needs to be suitable for patients with Brunnstrom stages 1-4 (Brunnstrom, 1966).

\section{Scenario Analysis}

Scenario analysis was based on on-site observations and the literature, and these were used to establish four stroke patient archetypes (see Table 1). The four stroke patient archetypes were constructed to allow the scenario analysis to be carried out based on the results obtained during the on-site observations. These representative archetypes ought to be able to account for the behavior of four completely different patient types. We then used a situational narrative approach to explore the usability problems with respect to the aforementioned stroke archetypes and to propose potential solutions.

\section{Design Conditions}

Most rehabilitation devices, such as push-pull boxes, pulleys/ tackle, and stacking require autonomic exertion by the patient. This study, however, is primarily concerned with stroke patients who have limited functionality in term of this level of autonomy. We proposed a rehabilitation device that not only maintains the motor activity and the range of motion of the upper limb but also induces better scapula motion than BATRAC-like devices. Observations, interviews, the Persona method, and situational narratives were used to explore potential usage problems. The results raised the following design specifications that would be required to fulfill the patients' rehabilitation needs: (1) an auxiliary arm support, (2) an auxiliary handle, (3) active machine movement, (4) bilateral mirrored action, (5) a fixed path, and (6) an emergency cutoff.

\section{Proposed Design}

Based on the design specifications described above, we developed a prototype (Figure 2) that was $1,180 \mathrm{~mm} \times 440 \mathrm{~mm} \times 300 \mathrm{~mm}$ in size and had a shell constructed of acrylonitrile butadiene styrene (ABS). The control module featured an on/off switch, a knob for adjusting the speed, and an emergency cut off switch.

The patient's hands grip each of the handles. An electric bandage was applied to assist grip when the hand on the affected side was unstable with respect to griping the handle. The movement of both arms was driven by a servo motor operating at $6,500 \mathrm{rpm}$, with a force of $0.28 \mathrm{~N}$. The force was then transmitted via two gear ratios (1:5 and 1:3). As the device is decelerated by these gears, the torque is increased to $12.6 \mathrm{~N}$, which corresponds to an output force of $420 \mathrm{~N}$.

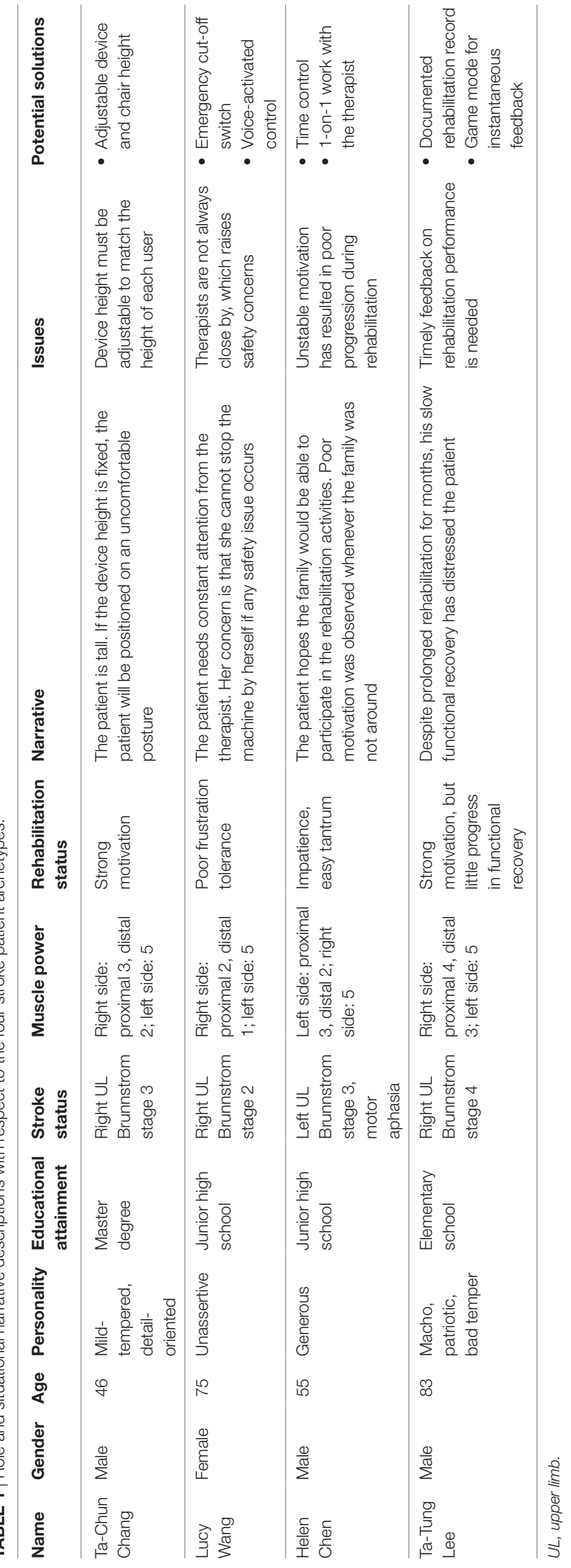




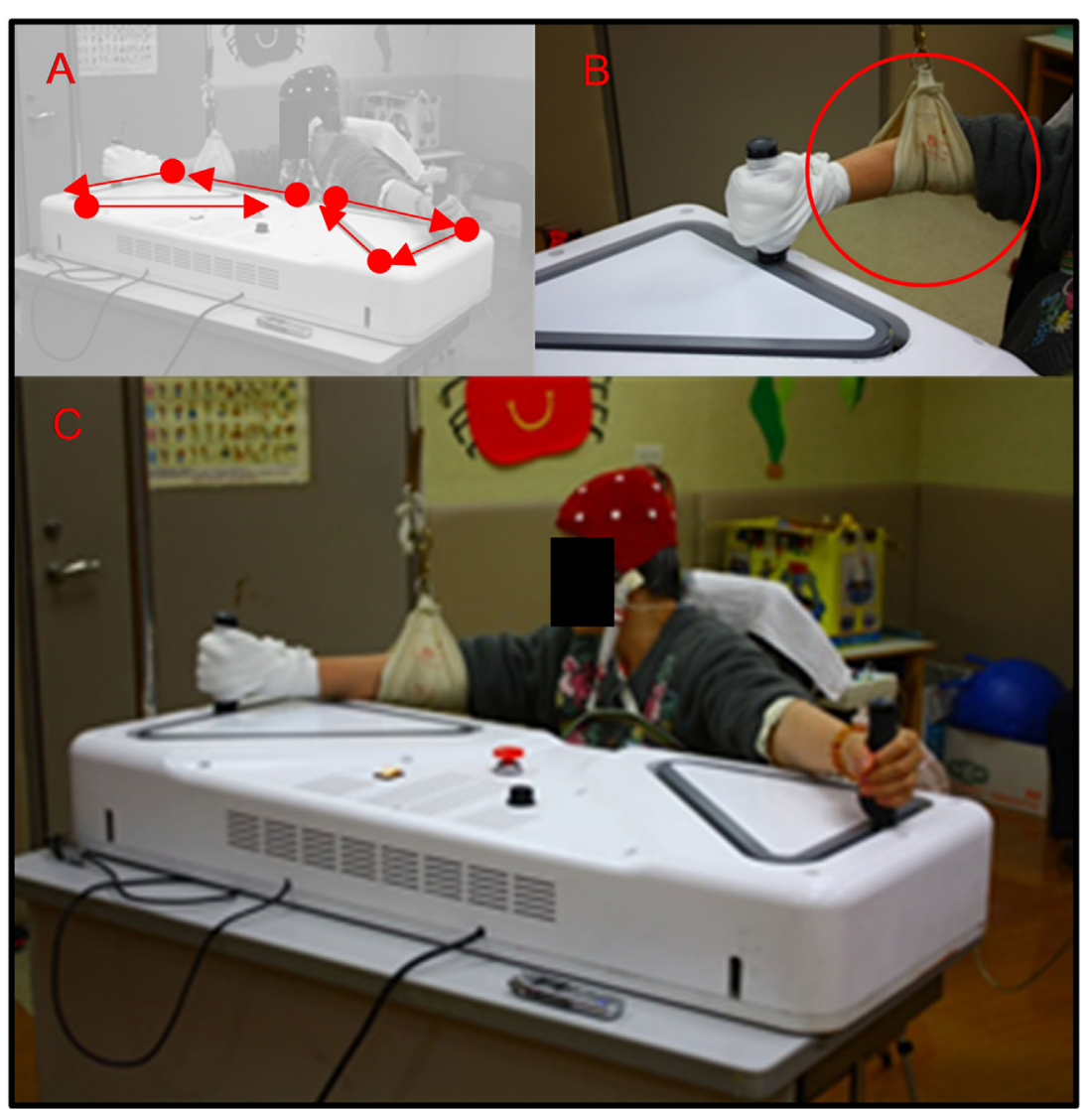

FIGURE 2 | (A) A patient performed bilateral diagonal movements using the device; (B) due to weakness of right upper limb, the patient's grip was assisted with an elastic bandage, and the patient's elbow was support by a sling; (C) the application scenario.

A gear-pulley-belt system is used to drive the handles, because rails cannot be used to form a triangular track (Figures 2A-C). Each upper limb was moved along a triangular pathway on the horizontal plane and the movements of the two hands are perfectly synchronized, yielding perfectly mirrored motion of both upper limbs. Specifically, the right and left upper limbs are guided to move clockwise and counterclockwise, respectively. The speed of motion is adjustable and can be set to range from 20 to $300 \mathrm{~mm} / \mathrm{s}$. During the following assessment periods, the speed was fixed at $20 \mathrm{~mm} / \mathrm{s}$.

\section{Stage 2: Prototype Usability Assessment The Pretest}

Five subjects (one male and four females) participated in the pretest during the recruitment period from 01-08-2013 to 31-012014 (Figure 3). The subjects were healthy individuals with ages ranging from 23 to 41 years; they had educational attainment levels ranging from high school to postgraduate studies. None had any previous experience using rehabilitation equipment.

The heights of the chair and the table were adjusted to match the height of the individual subjects. During the operation of the device, we observed that the handle rotates during operation, which may cause discomfort to some subjects who may seek to continuously correct their grip on the handle. Later, we asked participants to grip the handle tightly and they were not to allow to constantly adjust their grip.

During the pretest, the researchers adjusted the device speed three times. Subject 1 indicated that she felt more comfortable at higher speeds, while the others did not express a preference. Generally speaking, subjects felt that the activity was more tedious at slower speeds, but slower speeds are theoretically safer for stroke patients. The therapists suggested that the speed along the hypotenuse part of the movement track should be increased, allowing the subjects to stop in certain positions, and thus spending more time in a stretched positions. Another suggestion was to allow for the direction of the path to be reversed.

The SUS questionnaire score for the subjects ranged from 67.5 to 100 , with an average score of $83.5 \pm 13.1$, indicating a high degree of acceptance (between "good" and "excellent" on the overall usability scale). Categorizing the SUS questionnaire items in relation to usability (Q1, Q2, Q3, Q5, Q6, Q7, Q8, Q9) and learning $(\mathrm{Q} 4, \mathrm{Q} 10)$ domains, this showed an average usability score of $85.0 \pm 14.2$ and an average learning score of $77.5 \pm 9.7$. This indicates that the device usability domain is more effective than its learning counterpart (see Table 2). Moreover, reliability analysis produces a Cronbach's $\alpha$ value of 0.96 , indicating a very high degree of internal consistency (Cronbach, 1951). 


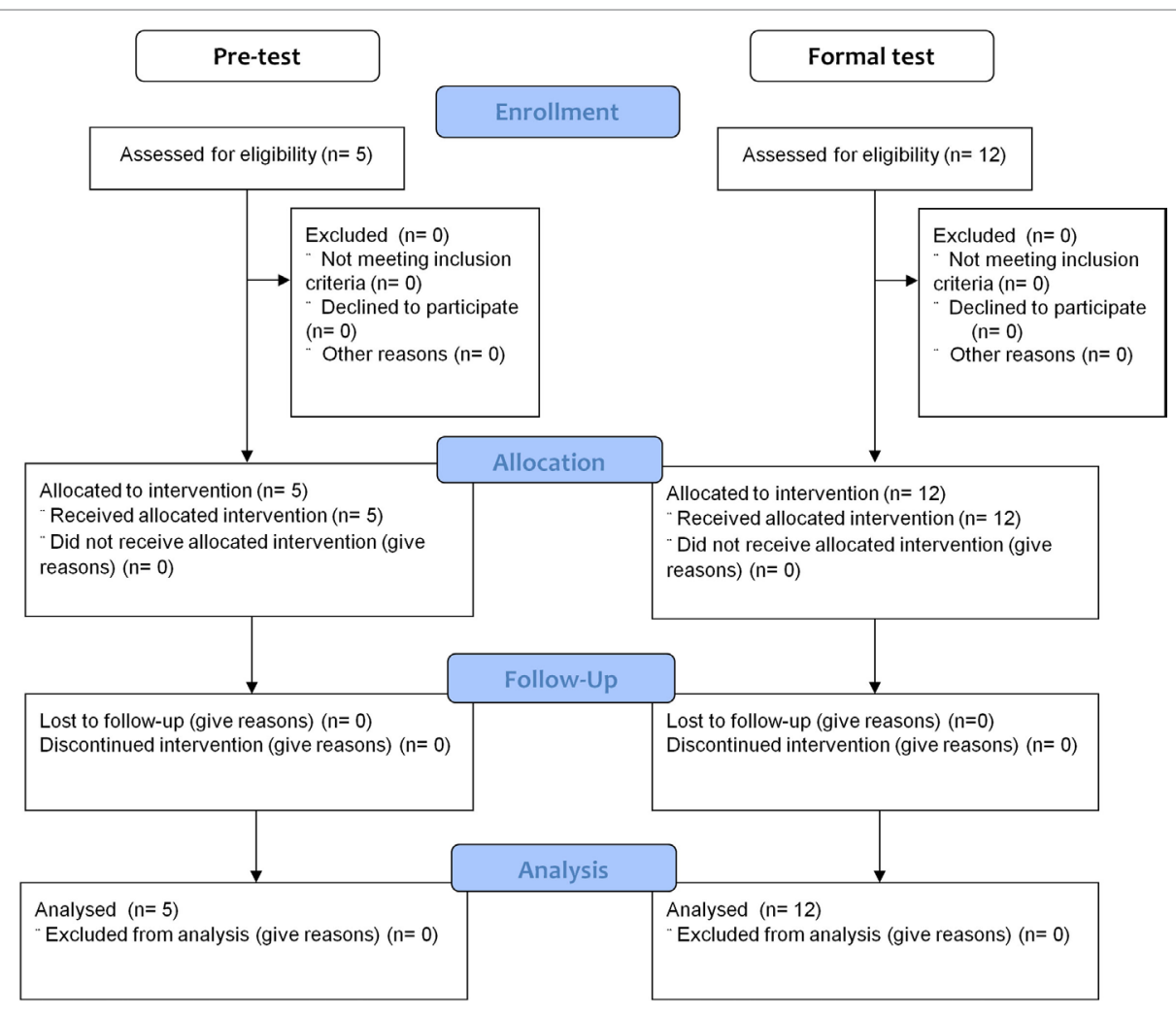

FIGURE 3 | The flowchart for subject enrollment, assignment, allocation, follow-up, and analysis for the pretest and formal test.

TABLE 2 | Total system usability scale (SUS), usability, and learning scores obtained in the pretest.

\begin{tabular}{lccc}
\hline Item & Total SUS score & Usability score & Learning score \\
\hline Score & $83.5 \pm 13.1$ & $85.0 \pm 14.2$ & $77.5 \pm 9.7$ \\
\hline
\end{tabular}

\section{The Formal Test}

As shown in Table 3, during the recruitment period from 01-02-2014 to 31-01-2016, 12 subjects (five males, seven females), with their age ranging from 25 to 60 years, and their educational attainment levels ranging from elementary school to graduate level, were recruited for the formal test (Figure 3). Two of the subjects had previous experience using robotic rehabilitation equipment systems. Table 4 summarizes the issues raised by the subjects. In total, four issues were raised and the following solutions were created to solve these issues. First, the hand on the paralyzed side was often unable to maintain a grip on the handle. To accommodate this, we applied an elastic bandage that provided additional hand grip support. Second, the height of the instrument was too high for most subjects. To solve this difficulty, we adjusted the chair and table to avoid the problem. Third, the subject's trunk was forced to bend forward when the motion reached to most forward corner, a problem that was caused by incorrect positioning and the distance between the instrument and subject along the anterior-posterior axis together with inadequate trunk support. As a result, we repositioned the instrument
TABLE 3 | Biographical data of the subjects participating the formal test.

\begin{tabular}{lclccc}
\hline $\begin{array}{l}\text { Subject } \\
\text { number }\end{array}$ & Gender & Background & Age & $\begin{array}{c}\text { Educational } \\
\text { attainment }^{\mathbf{a}}\end{array}$ & $\begin{array}{c}\text { Experience } \\
\text { using robotic } \\
\text { rehabilitation } \\
\text { equipment }\end{array}$ \\
\hline 01 & F & Patient & 60 & 3 & No \\
02 & M & Therapist & 28 & 4 & No \\
03 & F & Therapist & 27 & 5 & No \\
04 & F & Therapist & 36 & 4 & No \\
05 & F & Caregiver & 25 & 4 & No \\
06 & M & Patient & 45 & 2 & No \\
07 & F & Caregiver & 34 & 4 & Yes \\
08 & M & Patient & 45 & 4 & No \\
09 & F & Caregiver & 32 & 1 & No \\
10 & M & Patient & 49 & 3 & No \\
11 & F & Caregiver & 56 & 2 & \\
12 & m & Therapist & 56 & 4 &
\end{tabular}

M, male; F, female.

a1-elementary school; 2-junior high school; 3-senior high school; 4-university;

5-graduate school.

taking in to consideration the subject's upper limb length. Finally, the belt that guides the movement was quite loose causing the handle to wiggle intermittently. In this case, the tension of the belt was adjusted to minimize this problem.

Table 5 shows that an average SUS score of $71.8 \pm 11.9$ was obtained during the formal test. As was the case previously, the total usability score $(73.7 \pm 12.3)$ was higher than the learning 
TABLE 4 | Issues raised by the subjects in the formal test.

\begin{tabular}{|c|c|}
\hline Item & Solution \\
\hline Handle rotates during operation & $\begin{array}{l}\text { Application of an elastic bandage to } \\
\text { provide additional position support }\end{array}$ \\
\hline $\begin{array}{l}\text { The machine is positioned too high for } \\
\text { most subjects }\end{array}$ & $\begin{array}{l}\text { Adjusted the chair and table to avoid } \\
\text { this problem }\end{array}$ \\
\hline $\begin{array}{l}\text { When the motion reaches the top } \\
\text { corner of the triangle, the subject is } \\
\text { forced to lean forward to complete the } \\
\text { action, and then gradually recover }\end{array}$ & $\begin{array}{l}\text { Re-position the instrument taking in } \\
\text { to consideration the subject's upper } \\
\text { limb length }\end{array}$ \\
\hline $\begin{array}{l}\text { The left side belt is loose, causing the } \\
\text { handle to wiggle laterally }\end{array}$ & $\begin{array}{l}\text { The tension of the belt was adjusted to } \\
\text { minimize this problem }\end{array}$ \\
\hline
\end{tabular}

TABLE 5 | Total system usability scale (SUS), usability, and learning scores obtained in the formal test.

\begin{tabular}{lccc}
\hline Item & SUS score & Usability score & Learning score \\
\hline Score & $71.8 \pm 11.9$ & $73.7 \pm 12.3$ & $64.6 \pm 7.8$ \\
\hline
\end{tabular}

TABLE 6 | Score for each system usability scale item.

\begin{tabular}{|c|c|c|}
\hline Item & Content & Score \\
\hline Q1 & $\begin{array}{l}\text { I think I would like to use the stroke rehabilitation device } \\
\text { often }\end{array}$ & $3.67 \pm 0.78$ \\
\hline Q2 & I think the stroke rehabilitation device is difficult to use & $4.00 \pm 0.43^{a}$ \\
\hline Q3 & I think the stroke rehabilitation device is easy to use & $4.33 \pm 0.49$ \\
\hline Q4 & $\begin{array}{l}\text { I required technical assistance to use the stroke } \\
\text { rehabilitation device }\end{array}$ & $3.17 \pm 1.11^{\mathrm{a}}$ \\
\hline Q5 & $\begin{array}{l}\text { I think the functionalities of the stroke rehabilitation device } \\
\text { are well integrated }\end{array}$ & $3.58 \pm 1.08$ \\
\hline Q6 & $\begin{array}{l}\text { I think the functionality of the stroke rehabilitation device } \\
\text { are not organized }\end{array}$ & $3.58 \pm 0.90^{a}$ \\
\hline Q7 & $\begin{array}{l}\text { I think most users will be learn to use the stroke } \\
\text { rehabilitation device quickly }\end{array}$ & $4.08 \pm 0.79$ \\
\hline Q8 & $\begin{array}{l}\text { I think most of users will have difficulties learning to use the } \\
\text { stroke rehabilitation device }\end{array}$ & $4.25 \pm 0.45^{a}$ \\
\hline Q9 & I am confident when using the stroke rehabilitation device & $4.08 \pm 0.79$ \\
\hline Q10 & $\begin{array}{l}\text { I may need to learn more background information before } \\
\text { I am able to use the stroke rehabilitation device }\end{array}$ & $4.00 \pm 0.85^{a}$ \\
\hline
\end{tabular}

${ }^{a}$ Recoded by Eq. 1.

score $(64.6 \pm 7.8)$. The SUS scores for each subject, with scores that ranged from 42.5 to 92.5 , indicate usability between "Fair" and "Good." The level of acceptance is high, indicating that the patients and therapists were generally satisfied with the performance of the device.

The average score for each item ranges from 3.17 to 4.33 (Table 6). Items Q2, Q4, Q8, and Q10 were negative questions, and thus their scores are recoded before calculation, using the equation:

$$
\text { Recoded score }=5-\text { original score }
$$

After the recoding, item Q3 (Question "I think this new stroke rehabilitation system is easy to use") had the highest score, while item Q4 (Question "I required technical assistance to use this new stroke rehabilitation system") had the lowest score.
Reliability analysis shows a Cronbach's $\alpha$ value of 0.803 for the SUS scores, again indicating good internal consistency. We further applied the single-sample $t$-test to compare our score to the average SUS score obtained by Sauro (2013) who used a population of 500 different products that have been sold in the market (a score of 68) and found no significant difference $[t(1)=1.13$, $p=0.282]$. The results indicate that the usability of our design is not statistically different from those of most market products.

\section{DISCUSSION}

The present study demonstrates the development process of a novel rehabilitation device for stroke patients, which uses the Tseng's development method (Tseng, 2013). The development process involved the active participation of stroke patients and their therapists, ensuring that there was an understanding of the patients' needs so that that the product was approached from the point of view of the actual usage conditions during the early development stages. Indeed, even though a literature review and clinical observation can provide insight into basic product design concepts, the developer may still suffer from insufficient understanding of the dynamic interaction between the patient's physical movement limitations and the robotic device. In this context, the observation stage was able to reveal potential problems that allowed adjustments to be made to design fundamentals. Furthermore, interviews with the therapists, patients, and caregivers further validated the clinical usability of the product. Finally, the creation of subject archetypes and the situational use of narrative methods helped to raise other potential issues and various design modifications were incorporated into the concept sketches and mock-up, allowing inclusion in the engineering specifications.

\section{Strengths}

The significance of this study is that it provides development insight and subsequent usability validation for robotic-assistance rehabilitation devices targeting patients with neurological disorders. The SUS usability questionnaire shows good internal consistency. One important finding is that the pretest SUS score is relatively higher than the formal test core, a difference that might be accounted for by the fact that the pretest was applied to healthy subjects, while the formal test used the actual target subject group, including patients, therapists, and caregivers. Another finding is that usability scores are higher than learning scores, indicating that, if an effective learning method can been applied, then minor usability problems can be overcome. Finally, the usability score was comparable to those obtained from products already on the market, indicating that the rehabilitation device has reach considerable utility even though several minor issues still need to be addressed before it can be applied during advanced clinical research.

During the experimental process, the patients provided positive feedback and indicated they would be willing to use the device for rehabilitation. It is worth noting that the three therapists offered usability scores of $62.5,72.5$, and 42.5 . The therapists have occupational knowledge and this gives them particular insights into the actual rehabilitation status of patients and future research 
should target in-depth interviews with therapists in order to solicit suggestions for improvements to the device. Patients with experience using motorized rehabilitation equipment gave scores of 75.0 and 77.5 , indicating a positive response among experienced patients. In the formal test, the lowest scores came from one therapist who raised several concerns. The therapist specifically suggested that the wrist should remain static during operation and indicated that a patient with Brunnstrom stages 1 or 2 would have trouble griping the handle.

\section{Weaknesses}

During the design process, we were unable to find an adjustableheight table that was able to adequately fulfill the users' needs and thus future work will address this issue. In addition, the ergonomics of the device need to be improved in order to allow it to fit a wider range of potential users. During operation, the two handles tend to rotate, indicating that there is a need for improvements in some details of the machine. We are developing new functionalities that will provide graded resistance levels, allow the user to control exercise duration, allow recording of the patient's behavioral performance, and bring about the incorporation of game-based interfaces. Finally, this experiment only involved a small number of participants and future studies will need to recruit a larger pool of individuals to be tested in order to evaluate the device's clinical usability, thus allowing the device to be more fully applied in the field and to maximize its therapeutic value.

\section{CONCLUSION}

The MirrorPath developed in this study is based on the bilateral movement and PNF theories and uses motor-driven motion to apply passive exercise to both of the patient's arms, thus both maintaining the range of motion of the shoulders and elbows and enhancing the coordination of both arms. During the course of the experiment, we found that this type of design is better suited to patients who lack voluntarily limb movement. The test results indicate that the device has a good degree of

\section{REFERENCES}

Brooke, J. (1996). SUS-A quick and dirty usability scale. Usability Eval. Ind. $189,4-7$.

Brooke, J. (2013). SUS: a retrospective. J. Usability Stud. 8, 29-40.

Brunnstrom, S. (1966). Motor testing procedures in hemiplegia: based on sequential recovery stages. Phys. Ther. 46, 357.

Burgar, C. G., Lum, P. S., Shor, P. C., and Van der Loos, H. F. M. (2000). Development of robots for rehabilitation therapy: the Palo Alto VA/Stanford experience. J. Rehabil. Res. Dev. 37, 663-674.

Cauraugh, J. H., Lodha, N., Naik, S. K., and Summers, J. J. (2010). Bilateral movement training and stroke motor recovery progress: a structured review and meta-analysis. Hum. Mov. Sci. 29, 853-870. doi:10.1016/j. humov.2009.09.004

Cronbach, L. J. (1951). Coefficient alpha and the internal structure of tests. Psychometrika 16, 297-334. doi:10.1007/BF02310555

Feys, H. M., De Weerdt, W. J., Selz, B. E., Cox Steck, G. A., Spichiger, R., Vereeck, L. E., et al. (1998). Effect of a therapeutic intervention for the hemiplegic upper limb in the acute phase after stroke: a single-blind, randomized, controlled multicenter trial. Stroke 29, 785-792. doi:10.1161/01.STR.29.4.785

Hesse, S., Schulte-Tigges, G., Konrad, M., Bardeleben, A., and Werner, C. (2003). Robot-assisted arm trainer for the passive and active practice of bilateral usability, and the observation of the users help to raise various issues that need further improvement. Products always require constant re-engineering and refinement in order to improve their practicality.

\section{ETHICS STATEMENT}

This study protocol was specifically approved by the Institutional Review Board of Chang Gung Medical Foundation with ID 1015038A3. The study has been registered in https://ClinicalTrials. gov with registration number NCT02698605.

\section{AUTHOR CONTRIBUTIONS}

Y-CP, J-LC, AW, and KT designed and conducted the experiment; $\mathrm{KT}$ analyzed these data; Y-CP and KT wrote the manuscript.

\section{FUNDING}

This work was supported in part by the Ministry of Science and Technology, Taiwan, ROC (https://www.most.gov.tw/en/ public) under Contract MOST 103-2628-H-182-001-MY2, MOST 104-2410-H-182-025-MY2, MOST 105-2632-H-182001, and MOST 106-2218-E-182-001 by the Chang Gung Medical Foundation (https://www.cgmh.org.tw/eng2002/index. asp) (Fund No. CMRPG 5D0006, CMRPG 5D0061, CMRPD 3E0371, CMRPG 5D01061, CMRPG 3E1721, CMRPG 3C0463, CMRPD3E0373, and CMRPD2F0211), and by the Healthy Aging Research Center/Chang Gung University (http://harc.cgu. edu.tw/bin/home.php?Lang=en) (Fund No. EMRPD1G0221, CMRPD1B0331, CMRPD1B0332). For the Chang Gung Medical Foundation grants, the CMRPG5D006, CMRPG 5D00061, CMRPD 3E0371, and CMRPD2F0211 grants provide personnel and consumables and the remaining three provide personnel support for data analysis. The funders had no role in study design, data collection and analysis, decision to publish, or preparation of the manuscript. The researchers also thank Ms. Yu-Fang Yeh for her early contributions on the manuscript.

forearm and wrist movements in hemiparetic subjects. Arch. Phys. Med. Rehabil. 84, 915-920. doi:10.1016/S0003-9993(02)04954-7

Krebs, H. I., Hogan, N., Aisen, M. L., and Volpe, B. T. (1998). Robot-aided neurorehabilitation. IEEE Trans. Rehabil. Eng. 6, 75-87. doi:10.1109/86.662623

Kwakkel, G., Kollen, B. J., and Krebs, H. I. (2008). Effects of robot-assisted therapy on upper limb recovery after stroke: a systematic review. Neurorehabil. Neural Repair 22, 111. doi:10.1177/1545968307305457

Kwakkel, G., Kollen, B. J., and Wagenaar, R. C. (1999). Therapy impact on functional recovery in stroke rehabilitation: a critical review of the literature. Physiotherapy 85, 377-391. doi:10.1016/S0031-9406(05)67198-2

Lee, M., Rittenhouse, M., and Abdullah, H. A. (2005). Design issues for therapeutic robot systems: results from a survey of physiotherapists. J. Intell. Robot. Syst. 42, 239-252. doi:10.1007/s10846-004-7194-y

Lewis, J. R., and Sauro, J. (2009). "The factor structure of the system usability scale," in Human Centered Design, ed. M. Kurosu (Berlin, Heidelberg: Springer), 94-103.

Lo, A. C., Guarino, P. D., Richards, L. G., Haselkorn, J. K., Wittenberg, G. F., Federman, D. G., et al. (2010). Robot-assisted therapy for long-term upperlimb impairment after stroke. N. Engl. J. Med. 362, 1772-1783. doi:10.1056/ NEJMoa0911341

Lu, E. C., Wang, R. H., Hebert, D., Boger, J., Galea, M. P., and Mihailidis, A. (2011). The development of an upper limb stroke rehabilitation robot: identification 
of clinical practices and design requirements through a survey of therapists. Disabil. Rehabil. Assist. Technol. 6, 420-431. doi:10.3109/17483107.2010.544370

Mackay, J., Mensah, G. A., Mendis, S., and Greenlund, K. (2004). The Atlas of Heart Disease and Stroke. Geneva: World Health Organization.

Sauro, J. (2013). 10 Things to Know About the System Usability Scale (SUS). Available at: http://www.measuringusability.com/blog/10-things-SUS.php

Sharman, M. J., Cresswell, A. G., and Riek, S. (2006). Proprioceptive neuromuscular facilitation stretching. Sports Med. 36, 929-939. doi:10.2165/00007256200636110-00002

Stoykov, M. E., Lewis, G. N., and Corcos, D. M. (2009). Comparison of bilateral and unilateral training for upper extremity hemiparesis in stroke. Neurorehabil. Neural Repair 23, 945-953. doi:10.1177/1545968309338190

Timmermans, A. A. A., Seelen, H. A. M., Willmann, R. D., and Kingma, H. (2009). Technology-assisted training of arm-hand skills in stroke: concepts on reacquisition of motor control and therapist guidelines for rehabilitation technology design. J. Neuroeng. Rehabil. 6, 1. doi:10.1186/1743-0003-6-1
Tseng, K. C. (2013). "An IPDD approach for systematic innovation of products, processes, and services: a case study on the development of a healthcare management system," in The 5th IASDR World Conference on Design Research (Tokyo: IASDR).

Conflict of Interest Statement: The authors declare that the research was conducted in the absence of any commercial or financial relationships that could be construed as a potential conflict of interest.

Copyright (c) 2017 Pei, Chen, Wong and Tseng. This is an open-access article distributed under the terms of the Creative Commons Attribution License (CC BY). The use, distribution or reproduction in other forums is permitted, provided the original author(s) or licensor are credited and that the original publication in this journal is cited, in accordance with accepted academic practice. No use, distribution or reproduction is permitted which does not comply with these terms. 